\title{
Did depressive symptoms affect recognition of emotional prosody in Parkinson's disease?
}

\author{
Adriana Vélez Feijó' \\ Carlos RM Rieder ${ }^{3}$ \\ Márcia LF Chaves ${ }^{2}$ \\ 'Medical Sciences Post-Graduate \\ Course; ${ }^{2}$ Internal Medicine \\ Department, School of Medicine, \\ Universidade Federal do Rio \\ Grande do Sul, Porto Alegre, RS, \\ Brazil; ${ }^{3}$ Movement Disorders Clinic \\ Coordinator, Hospital de Clínicas de \\ Porto Alegre, Porto Alegre, RS, Brazil
}

\begin{abstract}
Objective: Evaluate the influence of depressive symptoms on the recognition of emotional prosody in Parkinson's disease (PD) patients, and identify types of emotion on spoken sentences.

Methods: Thirty-five PD patients and 65 normal participants were studied. Dementia was checked with the Mini Mental State Examination, Clinical Dementia Rating scale, and DSM IV. Recognition of emotional prosody was tested by asking subjects to listen to 12 recorded statements with neutral affective content that were read with a strong affective expression. Subjects had to recognize the correct emotion by one of four descriptors (angry, sad, cheerful, and neutral). The Beck Depression Inventory (BDI) was employed to rate depressive symptoms with the cutoff 14 .

Results: Total ratings of emotions correctly recognized by participants below and above the BDI cutoff were similar among PD patients and normal individuals. PD patients who correctly identified neutral and anger inflections presented higher rates of depressive symptoms ( $p=0.011$ and 0.044 , respectively). No significant differences were observed in the normal group.

Conclusions: Depression may modify some modalities of emotional prosody perception in $\mathrm{PD}$, by increasing the perception of non-pleasant emotions or lack of affection, such as anger or indifference.
\end{abstract}

Keywords: emotional prosody, Parkinson's disease, depression, emotion

\section{Introduction}

The nonverbal aspects of language have been defined as prosody, which transmits attitudinal, emotional, and similar information during communication. The prosodic style of conversation is essential to express the precise meaning and the affective context of what is trying to be said (Monrad-Krohn 1948). Emotional prosody is the ability to understand the affective or emotional tone of speech, which is believed to be a product of innate mechanisms, suggesting neural implementation of such processing (Adolphs et al 2002). There is evidence that recognition of emotion from prosody is accomplished similarly across different cultures (van Bezooijen et al 1983; Scherer et al 2001), and appears to emerge consistently by the first 6 years of life (Matsumoto and Kishimoto 1983). Depressed patients may present with an inability to memorize emotionally "enhancing" information due to an inability to access positive life events (Moore et al 1988). Many authors also consider the mood congruence hypothesis for depressed patients, supporting the preference to manifest and store negative information (Jorm and Henderson 1992).

$\mathrm{PD}(\mathrm{PD})$ is a neurodegenerative disorder associated primarily to degeneration of the dopamine-producing cells in the pars compacta of the substantia nigra (Bergman and Deuschl 2002). Cell loss in other pigmented brain stem nuclei, autonomic nuclei, and pyramidal cells in the presupplementary cortex also occurs (MacDonald and Halliday 2002). PD diagnosis is a clinical process based on the signs of resting tremor, bradykinesia 
or slowness of movement, rigidity, and loss of postural reflexes (Sethi 2002). The major problems that patients experience after 5 years of treatment are fluctuations (both motor and nonmotor), dyskinesias, and behavioral or cognitive changes (Witjas et al 2002), all problems that negatively affect quality of life (Marras et al 2002). Major depression is one of the most common psychiatric disturbances observed in PD patients (20\%-40\%), and may precede the diagnosis of PD (Lieberman 2006). Depression is the most significant predictor of quality of life (Lauterbach 2004). Additionally, PD may affect olfaction and verbal communication.

Olfaction is frequently impaired (Ward et al 1983), and the olfaction testing should be considered to differentiate PD from progressive supranuclear palsy and corticobasal degeneration, but not PD from multisystemic atrophy (Suchowersky et al 2006). Functional impairment of articulation, phonation, prosody and respiration may also occur (Logeman et al 1978; Darkins et al 1988). Speech disorder can be frequent as $89 \%$ in PD and often impacts on quality of life (Aronson 1990). Impairment of emotional prosody has been suggested to be present in PD patients (Borod et al 1990; Caekebeke et al 1991; Pell 1996).

The aim of this study was to evaluate the influence of depressive symptoms on the recognition of emotional prosody in PD patients. Types of emotion expressed on spoken sentences (anger, sadness, cheerfulness, and neutral) were also analyzed according to the presence of depressive symptoms.

\section{Methods}

Thirty-five PD patients and 65 normal subjects were crosssectionally analyzed over a 10-month period. The main outcome was the recognition of emotional prosody.

\section{Subjects}

Thirty-five PD outpatients who fulfilled inclusion criteria and were diagnosed and followed at the Movement Disorder Outpatient Clinic of a large University hospital in Porto Alegre (RS, Brazil) were consecutively enrolled in the study. An age- and sex-balanced community group $(n=65)$ without neurological findings or disorders affecting the central nervous system was selected as the comparison group for the study. Age varied from 50 to 80 years.

Inclusion criteria were diagnosis of idiopathic PD on the basis of clinical evaluation and response to levodopa or dopamine agonists (Gibb and Lees 1988; Hughes et al 2002), no hearing impairment above $40 \mathrm{~dB}$ (checked by hearing tests), no musical training, native Portuguese speaking, and residing in the state of Rio Grande do Sul.
Exclusion criteria were cerebrovascular disorders, use of antidepressants, psychiatric comorbidities (dementia, psychosis, and anxiety), previous alcohol or drug abuse and brain trauma. DSM IV criteria were applied for psychiatric comorbidities, including dementia. The Mini Mental State Examination was used to evaluate cognitive status (Folstein et al 1975; Miyasaki et al 2006). Patients were all nondemented, as evaluated by the Clinical Dementia Rating scale (CDR) (Hughes et al 1982; Miyasaki et al 2006; Chaves et al 2007); they were oriented and fully capable of themselves, and were reported by their spouses or caregivers to not have, or only very mild, impairment in judgment, problem solving, community affairs and life at home. The Schwab and England scale for activities of daily living was used for this purpose (Schwab and England 1969).

Degree of impairment due to PD was quantified using the Hoehn-Yahr scale (Hoehn and Yarh 1967). All PD patients were on dopaminergic replacement therapy (all 35 were taking levodopa plus carbidopa: mean oral dosage $\mathrm{mg} /$ day $=563.0 \pm 242.8$ ). None of the patients were taking anticholinergics or amantadine at the time of the study. All patients were tested during their optimally medicated ("'on'") state. None of the PD patients presented severe dyskinesia or resting tremor that could have interfered with testing. No patient was also receiving antidepressants.

Sample size was based on the following parameters: significance of 5\% (alpha error), power of 20\% (beta error), and frequency of dysprosody of $30 \%$ among PD patients.

\section{Main outcome measure}

\section{Recognition of emotional prosody}

A semantically neutral Portuguese sentence (eg, the table is made of wood) was spoken by a female voice for each of four different prosodic emotional descriptors: anger, sadness, happiness and neutral. Globally, subjects heard the recording play semantically neutral sentences 12 times, with three statements for each descriptor. Patients and normal participants could study the modalities of emotional prosody and answers given to the examiner for each presentation were 0 ("not at all") or 1 ("very much") with respect to the labels "angry", "happy", "sad", and "neutral", according to the protocol of Breitenstein and colleagues (2001). All participants were tested individually in a quiet room. The sentences were recorded in a soundproof chamber by two experienced speech pathologists.

\section{Controlled variables}

\section{Ratings of depressive symptoms}

Depressive symptoms were rated by the Beck Depression Inventory (BDI; Beck 1987). The cutoff of 14 (Leentjens et al 
2000) was applied to identify participants with presence of mild symptoms of depression. This standard clinical depression scale contains more questions about cognitive aspects of depression than somatic states and symptoms; therefore, we avoided the overlap of somatic features of depression and those related to PD.

\section{Ethical aspects}

The study was approved by the Ethics Committee for Medical Research of the Hospital where it took place. All participants signed an informed consent before being enrolled into the study.

\section{Statistical analysis}

Data evaluation

Group comparisons that were not normally distributed (mostly count data) were analyzed using nonparametric evaluation procedures (Kruskal-Wallis analyses, Mann-Whitney U-test, and Wilcoxon matched-pairs test). Parametric variables were analyzed by Student's t-test for independent samples. Categorical data were studied using contingence tables and chi-square association test (Yates correction or Fisher exact when necessary).

\section{Results}

Demographical and clinical data: The two groups did not show differences in terms of age, educational level, sex distribution, and social background (Table 1). 53\% of the whole sample completed the intermediate school. Parkinson patients presented higher scores in the BDI than normal subjects $(p=0.001)$. Within PD patients, $40 \%(n=14)$ presented depressive symptoms $($ Beck $\geq 14)$, while just $9 \%(n=6)$ of the normal individuals presented (chi-square $=13.46$; $p<0.001)$.

Frequency of types of emotion correctly recognized: Table 2 displays the frequency of participants who correctly recognized most of the emotional prosody sentences from the study groups. Among PD patients, anger was correctly recognized by $60 \%(p=0.011)$ and only by $32 \%$ of normal participants. Cheerful was correctly identified by almost half of the normal group (46\%) but only by $17 \%$ of the Parkinson's group $(p=0.030)$. Sadness was recognized by $54 \%$ of PD patients and $71 \%$ of normal participants $(p=0.125)$. The neutral intonation was recognized by $69 \%$ of normal individuals and $57 \%$ of PD patients $(p=0.650)$.

Relation of depressive symptoms with recognition of emotional prosody: The total score of the emotions correctly recognized by participants from PD and normal groups with BDI $<14$ were similar to scores presented by individuals with BDI $\geq 14$ (Table 3).

Of the PD patients, those who correctly identified neutral $(p=0.026)$ and anger $(p=0.047)$ inflections presented higher rates of depressive symptoms (Table 4 ).

No statistically significant differences were observed on rates of symptoms of depression related to recognize, correctly or incorrectly, different types of emotional prosody for normal participants (Table 5).

\section{Discussion}

We evaluated the relation of depressive symptoms and the recognition of emotional prosody in nondemented PD patients and normal subjects. The two groups presented similar demographic characteristics. PD patients showed higher rates of depressive symptoms (BDI) than normal

Table I Demographical and clinical data of the sample

\begin{tabular}{|c|c|c|c|}
\hline & Parkinson disease $(n=35)$ & Normal participants $(n=65)$ & $p$ value \\
\hline Age* & $63.74 \pm 10.20$ & $63.62 \pm 9.06$ & 0.99 \\
\hline \multicolumn{4}{|l|}{ Sex $* *$} \\
\hline Male & $18(5 \mid .4 \%)$ & $30(46.2 \%)$ & \\
\hline Female & $17(48.6 \%)$ & $35(53.8 \%)$ & 0.768 \\
\hline \multicolumn{4}{|l|}{ Education** } \\
\hline Intermediate school & $23(65.7 \%)$ & $30(46.2 \%)$ & 0.131 \\
\hline High school & $10(28.6 \%)$ & $25(38.5 \%)$ & \\
\hline College/University & $2(5.7 \%)$ & $10(15.4 \%)$ & \\
\hline SRQ scores & $6.25 \pm 3.64$ & $2.71 \pm 2.11$ & 0.001 \\
\hline Beck Depression Inventory* & $|4.14 \pm| 0.5 \mid$ & $7.28 \pm 4.7$ & 0.001 \\
\hline Disease duration (years) & $6.94 \pm 4.17(3-17)$ & - & - \\
\hline \multicolumn{4}{|l|}{ Hoehn-Yahr scale } \\
\hline (Degree of impairment) & $2.06 \pm 0.94(I-4)$ & - & - \\
\hline Schwab and England scale - ADL & $78.00 \pm 19.60(30-100)$ & - & - \\
\hline
\end{tabular}

Notes: *Student's t test; ** $\chi^{2}$ test (with Yates correction or Fisher's Exact when needed). 
Table 2 Distribution (N,\%) of participants (Parkinson disease and normal participants) who correctly recognize each type of emotion verbally expressed on sentences

\begin{tabular}{llll}
\hline $\begin{array}{l}\text { Type } \\
\text { of emotion }\end{array}$ & $\begin{array}{l}\text { Parkinson disease } \\
(\boldsymbol{n}=35)\end{array}$ & $\begin{array}{l}\text { Normal participants } \\
(\boldsymbol{n}=65)\end{array}$ & $\boldsymbol{p}$ value* \\
\hline Anger & $21(60 \%)$ & $21(32 \%)$ & $0.01 \mathrm{I}$ \\
Sadness & $19(54 \%)$ & $46(71 \%)$ & 0.125 \\
Cheerful & $23(17 \%)$ & $30(46 \%)$ & 0.030 \\
Neutral & $26(74 \%)$ & $45(69 \%)$ & 0.650 \\
\hline
\end{tabular}

Notes: *Pearson chi-square with Fisher's Exact test.

subjects did. $40 \%$ of the patients presented symptoms above the cutoff (Leentjens et al 2000). The total score of emotional recognition was similar when we compared the PD patients and normal participants group. However, different types of emotion showed diverse pattern among the groups.

Although depression is often associated with PD, little is known about the influence of $\mathrm{PD}$ on recognition of emotional stimulus. Recognition of emotional prosody was similar between patients with and without depressive symptoms. This was also observed in the group of normal subjects, although fewer subjects $(9 \%)$ presented depression rates above cutoff.

Given the failure in finding a relationship between depression and overall prosody, we studied the modalities of emotional prosody. Analyzing types of emotion expressed on recorded samples, we observed that more PD patients $(60 \%)$ correctly recognized anger than normal participants did. On the other hand, the proportion of normal individuals that correctly recognized cheerfulness was higher (46\%) than the proportion of PD patients did (17\%).

Although we have not found influence of depressive symptoms on recognition of emotional expression overall, PD patients, who correctly identified anger and neutral inflections, presented higher rates of depressive symptoms. These results suggest that depression may modify some modalities of emotional perception. Emotional experience can be pleasant or unpleasant (eg, anger, sadness, and even lack of affection would be unpleasant). Depression in PD patients may increase the perception of unpleasant emotions such as anger and indifference. It is known that clinically depressed patients tend to see the outside world through the prism of their interior sadness (Jorm and Henderson 1992). Apathy and lack of interest are also frequently seen in depression and PD patients (Lieberman 2006), and these descriptors are intrinsically related to indifference. Happy subjects would produce more happy meanings of happy-neutral homophones than sad subjects, and sad subjects would produce more sad meanings of sad-neutral homophones than happy subjects (Halberstadt and Niedeenthal 2002). Therefore, PD depressive subjects would easily perceive expressions containing unpleasant feelings (Moore et al 1988). Degenerative disorders of the basal ganglia, as PD, damage the recognition of facial expression of anger (Scott et al 1984; Sprengelmeyer et al 1996), as well as comprehension of angry intonations (Breitenstein et al 1998). Recent data suggest involvement of the basal ganglia in emotional processing in general, but more specifically in complex emotional and social judgments (judgments of attractiveness of faces by ventral striatum) (Adolphs 2002).

Several studies with experimental animals have suggested that emotions such as fear or anger may facilitate overt behavior in parkinsonian states (Caekebeke et al 1991). For example, an akinetic parkinsonian state has been induced in rats following injection of hydroxydopamine, a dopaminergic toxin, into the lateral hypothalamic region of rat brains. When these akinetic rats were either put in with a group of cats or in an ice bath, they became active (Marshal et al 1976). Similar findings were demonstrated in cats with bilateral lesions to the lateral hypothalamus. Cats who exhibited akinesia following the lesions demonstrated increased spontaneous locomotion with externally stimulated arousal (eg, tail pinch), as well as increased eating, and orientation and response to somatosensory information. Thus, exposing rats to cats, and pinching the feet and tails of these experimental animals may increase arousal-activation in a manner similar to that seen in people who are experiencing fear or anger.

Although prior studies have demonstrated that stimuli that induce high levels of arousal-activation can increase overt behaviors in hypokinetic parkinsonian animals, the reason that subjects with PD increased their overt behavior more than normal subjects did, when conveying events that induced anger, is unknown. We suggest that this may be related to the

Table 3 Effect of depressive symptoms on recognition of emotional prosody (Kruskal-Wallis I-way ANOVA)

\begin{tabular}{|c|c|c|c|c|c|}
\hline & \multicolumn{2}{|l|}{ Parkinson disease } & \multicolumn{2}{|l|}{ Normal participants } & \multirow[t]{2}{*}{$p$ value } \\
\hline & Beck $<14(n=21)$ & Beck $\geq 14(n=14)$ & Beck $<$ I4 $(n=58)$ & Beck $\geq 14(n=07)$ & \\
\hline Total prosody recognition & $6.29 \pm 2.95(0-12)$ & $5.1 \pm 2.46(2-12)$ & $6.66 \pm 2.72(I-12)$ & $6.71 \pm 1.60(4-9)$ & 0.549 \\
\hline
\end{tabular}


Table 4 Mean \pm standard deviation of rates of depressive symptoms (Beck Depression Inventory): Parkinson's disease patients

\begin{tabular}{llll}
\hline \multirow{2}{*}{ Type of emotion } & Recognition & \multirow{2}{*}{$\boldsymbol{p}$ value* } \\
\cline { 2 - 3 } & Correct & Incorrect & \\
\hline Anger & $16.95 \pm 12.55(2 \mathrm{I})$ & $9.7 \mathrm{I} \pm 4.46(14)$ & 0.047 \\
Sadness & $14.16 \pm 11.00(19)$ & $13.94 \pm 10.57(16)$ & 0.952 \\
Cheerful & $9.50 \pm 4.24(08)$ & $15.41 \pm 11.62(27)$ & 0.171 \\
Neutral & $16.39 \pm 11.29(26)$ & $7.33 \pm 3.88(09)$ & 0.026 \\
\hline
\end{tabular}

Notes: *Student's t test for independent samples. Equal variances assumed.

higher perception of unpleasant stimulus by PD patients, and the higher the perception, the greater the reaction. Whether those differences actually represent major depression is not clear. Future research has to test this hypothesis.

Patients with PD often show an approximately 60\%-85\% loss of dopaminergic neurons in the substantia nigra and are typically characterized by tremor, rigidity, and bradykinesia (Goetz 2005). Degeneration of nigrostriatal dopaminergic pathways in patients with PD first affects the putamen, followed by the caudate nucleus. The basal ganglia has been implicated for emotion recognition (Gorelick and Ross 1987) for example, those with subcortical damage to this brain structure produced more pronounced deficits in emotion recognition than those without damage to the basal ganglia. The putamen, in addition to various frontal cortices, has been associated with recognizing happiness (Phillips et al 1998). Other brain structures associated with the basal ganglia have also been found to be involved in emotion recognition processes, including the amygdala, the insula, and the cerebellum (Mitchell et al 2003).

The present study presents some limitations. The number of patients, although supported by the sample calculation for dysprosody, may not be enough for all the analyses we carried out. On the other hand, most of the published papers on prosody and groups of patients showed samples even smaller than the present one and no sample size calculation to support the results. We could have performed other

Table 5 Mean \pm standard deviation of rates of depressive symptoms (Beck Depression Inventory):

Normal participants

\begin{tabular}{llll}
\hline Type of emotion & \multicolumn{2}{l}{ Recognition } & \multirow{2}{*}{ P value* } \\
\cline { 2 - 3 } & Correct & Incorrect & \\
\hline Anger & $7.29 \pm 5.15(44)$ & $7.00 \pm 4.06(21)$ & 0.818 \\
Sadness & $7.50 \pm 5.16(46)$ & $6.47 \pm 3.81(19)$ & 0.437 \\
Cheerful & $7.00 \pm 4.49(30)$ & $7.37 \pm 5.11(35)$ & 0.758 \\
Neutral & $6.84 \pm 5.10(45)$ & $8.00 \pm 4.03(20)$ & 0.374 \\
\hline
\end{tabular}

Notes: *Student's t test for independent samples. Equal variances assumed. testing together with the emotional prosody (ie, linguistic prosody) to correlate to other abilities. However, there was no reason to believe patients could have any prosodic impairment, unless emotionally toned, because they were cognitively normal. We performed the Mini Mental State Exam and the CDR scale to ascertain the cognitive status of both patients and normal participants. The main limitation of the study is the use of only one type of stimulus (recognition of emotion from recorded voice). The use of voice recordings could have drawn multiple prosodic cues and precluded clear results.

Finally, it may be important for caregivers and healthcare professionals to recognize that $\mathrm{PD}$ patients are more sensitive to external emotionally unpleasant stimuli.

\section{Disclosure}

The authors declare no conflicts of interest.

\section{References}

Adolphs R, Damasio H, Tranel D. 2002. Neural systems for recognition of emotional prosody: A 3-D lesion study. Emotion, 2:23-51.

Adolphs R. 2002. Neural systems for recognizing emotion. Curr Opin Neurobiol, 12:169-77.

Aronson AE. 1990. Clinical voice disorders. 3rd ed. New York: ThiemeStratton.

Beck AT. 1987. Beck Depression Inventory. San Antonio, TX: Psychological Corporation.

Bergman H, Deuschl G. 2002. Pathophysiology of Parkinson's disease: from clinical neurology to basic neuroscience and back. Mov Disord, 17:28-40.

Borod JC, Welkowits JMA, Brozgold AZ, et al. 1990. Parameters of emotional processing in neuropsychiatric disorders: Conceptual issues and battery of tests. J Commun Disord, 23:247-71.

Breitenstein C, Daum I, Ackermann H. 1998. Emotional processing following cortical and subcortical brain damage: Contribution of the fronto-striatal circuitry. Behav Neurology, 11:29-42.

Breteinstein C, Van Lancker D, Daum I, et al. 2001. Impaired perception of vocal emotions in parkinson's disease: influence of speech time processing and executive functioning. Brain Cogn, 45:277-314.

Caekebeke JFV, Jennekens-Schinkel A, van der Linden M, et al. 1991. The interpretation of dysprosody in patients with Parkinson's disease. $J$ Neurol Neurosurg Psychiatry, 54:145-48.

Chaves MLF, Camozzato AL, Godinho C, et al. 2007. Validity of the clinical dementia rating scale for the detection and staging of dementia in Brazilian patients. Alzheimer Dis Assoc Disord, 21:210-17.

Darkins AW, Fromkin VA, Benson DF. 1988. A characterization of the prosodic loss in Parkinson's disease. Brain Lang, 34:315-27.

Folstein M, Folstein S, McHugh P. 1975. Mini-mental state: A practical method for grading the cognitive state of patients for the clinician. J Psychiat Res, 12:189-98.

Gibb WR, Less AJ. 1988. The relevance of the Lewy body to the pathogenesis of idiopathic Parkinson's disease. J Neurol Neurosurg Psychiatry, $51: 745-52$.

Goetz CG. 2005. Parkinson Disease. In: Gilman S (ed.) MedLink Neurology. San Diego: MedLink Corporation [online]. Accessed August 05 2007 from http://www.medlink.com.

Gorelick PB, Ross ED.1987. The aprosodias: further functional-anatomical evidence for the organization of affective language in the right hemisphere. J Neurol Neurosurg Psychiatry, 50: 553-60. 
Halberstadt JB, Niedeenthal PM. 2002. Effects of emotions concepts on perceptual memory for emotional expressions. J Pers Soc Psychol, 81:857-98.

Hoehn MM, Yahr MD. 1967. Parkinsonism: onset, progression, and mortality. Neurology, 17:427-42.

Hughes AJ, Daniel SE, Ben-Shlomo Y, et al. 2002. The accuracy of the clinical diagnosis of parkinsonian syndromes in a specialist movement disorder service. Brain, 125:861-70.

Hughes CP, Berg L, Danziger WL, et al. 1982. A new clinical scale for the staging of dementia. Br J Psychiatr, 140:566-72.

Jorm AF, Henderson AS. 1992. Memory bias in depression: implications for risk factors studies relying on self-reports of exposure. Int J Methods Psychiat Res, 2:31-8.

Lauterbach EC. 2004. The neuropsychiatry of Parkinson's disease and related disorders. Psychiatr Clin North Am, 27:801-25.

Leentjens AF, Verhey FR, Luijckx GJ, et al. 2000. The validity of the Beck Depression Inventory as a screening and diagnostic instrument for depression in patients with Parkinson's disease. Mov Disord, 15:1221-4.

Lieberman A. 2006. Depression in Parkinson's disease - a review. Acta Neurol Scand, 113:1-8.

Logemann JA, Fischer HB, Boshes B, et al. 1978. Frequency and concurrence of vocal tract dysfunction in the speech of a large sample of Parkinson patients. $J$ Speech Hear Disord, 42:47-57.

MacDonald V, Halliday GM. 2002. Selective loss of pyramidal neurons in the pre-supplementary motor cortex in Parkinson's disease. Mov Disord, 17:1166-73.

Marras C, Lang A, Krahn M, et al. 2004. Quality of life in early Parkinson's disease: impact of dyskinesias and motor fluctuations. Mov Disord, 19:22-8.

Marras C, Rochon P, Lang AE. 2002. Predicting motor decline and disability in Parkinson's disease: a systematic review. Arch Neurol, 59:1724-8.

Marshal JF, Levitan D, Stricker EM. 1976. Activation-induced restoration of sensorimotor functions in rat with dopamine-depleting brain lesions. Comp Physiol Psychol, 90:536-46.

Matsumoto D, Kishimoto H. 1983. Developmental characteristics in judgments of emotion fron nonverbal vocal cues. Int J Intercult Relations, 7:415-24.

Mitchell RL, Elliott R, Barry M, et al. 2003. The neural response to emotional prosody, as revealed by functional magnetic resonance imaging. Neuropsychologia, 41:1410-21.
Miyasaki JM, Shannon K, Voon V, et al. 2006. Practice parameter: evaluation and treatment of depression, psychosis, and dementia in Parkinson disease (an evidence based review): report of the Quality Standards Subcommittee of the American Academy of Neurology. Neurology, 66:996-1002.

Monrad-Krohn GH. 1948. Dysprosody or altered "melody of language". Brain, 70:405-15.

Moore RG, Watts FN, Williams JM. 1988. The specificity of personal memories in depression. Br J Clin Psychol, 27:275-6.

Pell MD. 1996. On the receptive prosodic loss in Parkinson's disease. Cortex, 32: 693-704.

Phillips ML, Bullmore ET, Howard R, et al. 1998. Investigation of facial recognition memory and happy and sad facial expression perception: an fMRI study. Psychiatry Res, 83: 127-138.

Scherer KR, Banse R, Wallbott H. 2001. Emotion inferences from vocal expression correlate across language and cultures. J Cross-cult Psychol, 32:76-92.

Schwab RS, England AC. 1969. Projection technique for evaluating surgery in Parkinson's disease. In Gillingham FJ, Donaldson ML (eds). Third Symposium on Parkinson's Disease. Edinburgh: Livingstone, pp. 152-7.

Scott S, Caird FI, Williams B. 1984. Evidence for an apparent sensory speech disorder in Parkinson's disease. J Neurol Neurosurg Psychiatry, 47:840-3.

Sethi KD. 2002. Clinical aspects of Parkinson disease. Curr Opin Neurol, 15:457-60.

Sprengelmeyer R, Young AW, Calder AJ, et al. 1996. Loss of disgust Perception of faces and emotions in Huntington's disease. Brain, 119:1647-65.

Suchowersky O, Reich S, Perlmutter J, et al. 2006. Practice parameter: diagnosis and prognosis of new onset Parkinson disease (an evidencebased review) Report of the Quality Standards Subcommittee of the American Academy of Neurology. Neurology, 66:968-75.

van Bezooijen R, Otto SA, Heenan T. 1983. Recognition of vocal expressions of emotion - a three-nation study to identify universal characteristics. J Cross-cult Psychol, 14:387-406.

Ward CD, Hess WA, Calne DB. 1983. Olfactory impairment in Parkinson's disease. Neurology, 33:943-6.

Witjas T, Kaplan E, Azulay JP, et al. 2002. Nonmotor fluctuations in Parkinson's disease: frequent and disabling. Neurology, 59:408-13. 